\section{Program package STAP: A statistical appendix}

\author{
C. VAN DE WIJGAART \\ Technisch Centrum FSW, University of Amsterdam, \\ Amsterdam $1000 \mathrm{HE}$, The Netherlands
}

Like many other universities, the University of Amsterdam subscribes to program packages like SPSS and BMDP and also has a collection of self-developed or procured additional stand-alone programs. The latter category is often more up to date than the programs available in packages; however, these programs are often less user-friendly and are often incompatible with the control languages and data structure conventions of the packages.

Perhaps differently from other universities, the University of Amsterdam tried to find a solution by the construction of the package STAP.

STAP (an acronym for statistical appendix) is not meant to be a new "complete" package, but, rather, an appendix to SPSS, mainly by providing a number of more advanced statistical procedures in an SPSScompatible way. In the first version of STAP, which is now available, the number of statistical procedures is 13.

STAP uses an SPSS-compatible system file with an important modification: As most STAP procedures frequently use matrix input and output, the STAP system file is not only able to contain the raw data but offers matrix input, output, and storage as well. This way, matrices can be passed from one procedure to another within one set-up. Matrices and their functions are indicated by names and key words, minimizing the risk of administrative errors. Other advantages of the modified system file are that (1) other information, such as subfile group, the size of the matrix, and row and column labels, will automatically be stored and retrieved, (2) matrices can be used partially by selecting rows and columns, and (3) full machine precision will be retained.

The connection of STAP with SPSS has been realized as follows: STAP uses the same control language as SPSS and contains some data management functions known from SPSS. STAP can read and modify an SPSS system file, and the result can (generally) be read by SPSS.

The number of (raw) data management functions in STAP is small; STAP does not even offer the possibility of reading raw data. The user is expected to have done his data management in SPSS, including the creation of a system file. STAP can, without too much difficulty, be extended with new procedures.

STAP Procedures and Documentation. The STAP user's manual is contained in seven volumes, each dedicated to more or less related subjects or procedures.
Volume 1-Volume 1 includes an introduction to STAP (control language, relation STAP/SPSS, data definition and management), the STAP Matrix System Guide (the matrix system as an extension of the SPSS system file), a summary (of control cards, etc.), and an appendix (STAP execution within the CDC operating system).

Volume 2-Subprogram ASSOCIATION computes covariance, Pearson correlation, point biserial and phi coefficient, biserial phi, biserial correlation, tetrachoric, multiple, and Spearman's rank correlation. Subprogram DISTANCE computes Minkowski distances measures: the city block, euclidean, and dominance metric. Subprogram CLUSTER performs hierarchical cluster analyses according to (1) Johnson (the maximum and minimum method) and (2) Elshout (based on reciprocity and properties of the correlation coefficient).

Volume 3-Subprogram FACAN performs factor analysis as described in Jöreskog's (1963) dissertation. Subprogram FACAN SCORES provides estimation of factor scores according to Bartlett, the regression method, and a generalization of the Anderson-Rubin method (see Saris, de Pijper, \& Mulder, 1978). Subprogram PROCRUS ROTATE performs procrustean rotation of an orthogonal (factor) matrix toward a target matrix (oblique or orthogonal).

Volume 4--Subprograms MOKKEN SCALE and MOKKEN TEST perform stochastic cumulative scaling following Mokken's (1971) model (which is more general than the deterministic Guttman model).

Volume 5-Subprogram ITEM ANALYSIS computes the usual test statistics for multiple-choice or attitude and personality inventory tests, with optional information per subject. For attitude scales, the method of Lawshe and Harris is included.

Volume 6-Subprogram LINEAR COMB computes the (standardized) linear combinations of scores, which can be added to the system file. Subprogram SCATTER PLOT produces scatter diagrams of data points on a plotter or graphic display.

Volume 7-Subprogram NONPAR T-TEST performs Wilcoxon's rank-sum test and Ansari's test for independent samples and Wilcoxon's symmetry test and Spearman's rank correlation coefficient for paired samples. Subprogram NONPAR ONEWAY performs the Kruskal and Wallis rank-sum test for $k$ independent samples, Spjötvoll's contrast estimatc between k samples (Hollander \& Wolfe. 1973), Stecl's range test (Dunn, 1964), Jonckheere and Terpstra's trend test (Hollander \& Wolfe, 1973), and Doornbos' (Note 1) test for outliers.

The documentation of the procedures contains theoretical introductions, program descriptions, descriptions of the procedure cards, options and statistics, and sample output. 
STAP has been developed and documented at the Technisch Centrum of the Social Science Department of the University of Amsterdam, as commissioned by the Amsterdam Academic Computing Center, and incorporates programs submitted by the Free University and the Mathematical Center in Amsterdam.

The STAP package has been written in standard FORTRAN IV, augmented with CDC assembler when this was inevitable (e.g., in memory management and input/output). This makes the current version suitable for CDC Cyber installations only. The source code consists of 29,000 lines of FORTRAN code, 16,000 lines of assembler, and 30,000 lines of comment. The SPSS system file used is the one produced by SPSS. 6000 Version 8.

More information on STAP can be obtained from the author.

\section{REFERENCE NOTE}

1. Doornbos, R. Slippage tests (Mathematical Center Tracts, 15). Amsterdam: Mathematical Center, 1966.

\section{REFERENCES}

Dunn, O. J. Multiple comparison using rank sums. Technometrics, 1964, 6, 241.

Elshout, J. See: STAP user's manual. Amsterdam: Technisch Centrum, 1980.

Hollande R, M., \& Wolfe, D. A. Nonparametric statistical methods. New York: Wiley, 1973.

Johnson, S. C. Hierarchical clustering schemes. Psychometrika, $1967,32,241-254$.

JöreskoG, K. G. Statistical estimation in factor analysis. Stockholm: Almquist \& Wiksell, 1963.

Lawshe, C. H., \& Harris, D. H. The method of reciprocal averages in weighting personnel data. Educational and Psychological Measurement, 1958, 18, 2.

Mokken, R. J. A theory and procedure of scale analysis. Paris: Mouton, 1971.

Saris, W. E., de Pijper, M., \& Mulder, J. Optimal procedures for estimation of factor scores. Sociological Methods and Research, 1978, 7, 85-106.

(Accepted for publication June 8, 1981.) 